\title{
Space Nuclear Safety Program
}

March 1983

Compiled by

S. E. Bronisz

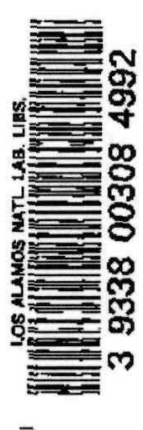




\section{DISCLAIMER}

This report was prepared as an account of work sponsored by an agency of the United States Government. Neither the United States Government nor any agency Thereof, nor any of their employees, makes any warranty, express or implied, or assumes any legal liability or responsibility for the accuracy, completeness, or usefulness of any information, apparatus, product, or process disclosed, or represents that its use would not infringe privately owned rights. Reference herein to any specific commercial product, process, or service by trade name, trademark, manufacturer, or otherwise does not necessarily constitute or imply its endorsement, recommendation, or favoring by the United States Government or any agency thereof. The views and opinions of authors expressed herein do not necessarily state or reflect those of the United States Government or any agency thereof. 


\section{DISCLAIMER}

Portions of this document may be illegible in electronic image products. Images are produced from the best available original document. 


\title{
SPACE NUCLEAR SAFETY PROGRAM
}

March 1983

\section{Compiled by}

S. E. Bronisz

\begin{abstract}
This technical monthly report covers studies related to the use of ${ }^{238} \mathrm{PuO}_{2}$ in radioisotope power systems carried out for the Office of Special Nuclear Projects of the US Department of Energy by Los Alamos National Laboratory. Most of the studies discussed here are ongoing. Results and conclusions described may change as the work continues.
\end{abstract}

\section{GENERAL-PURPOSE HEAT SOURCE (GPHS)}

\section{A. Phosphorus Effects Experiment (D. Pavone)}

The resuits of Auger Electron Spectroscopy (AES) analysis of the fracture surfaces of the as-received archive rings, the aged archive rings, the fueled iridium capsules, and the welds of the aged capsules are given in Table I. The aging exposures were 6 months at $1310^{\circ} \mathrm{C}$ in a FWPF* graphite container. The as-received samples contained grain-boundary concentrations of thorium at the levels expected for DOP-26 iridium. Carbon was not detected in these samples, but oxygen was observed in two of them. Some of the aged archive ring sampies showed substantial quantities of carbon and/or oxygen in addition to thorium, and one sample contained small quantities of sulfur and either boron or chlorine. The reason for the apparent pickup of carbon and oxygen by some samples and not others is unknown. Interestingly, this effect was also observed in the fueled-cup samples. In general, those archival rings that contained carbon or oxygen were from the fueled cups that contained the same elements, suggesting that the chemical make-up of

*Fineweave-Pierced Fabric 3-D carbon/carbon composite, a product of AVCO Systems Division, 201 Lowell St., Wilmington, MA 01887. the individual cup may influence its tendency to pick up impurities. Most of the fueled cups exhibited thorium depletion at the inner surface (fuel side). In some cups, thorium depletion at the outer surface (graphite side) was also detected. The welds of two capsules, PEF-48 and PEF-49, were severely depleted in thorium. It is possible that these analyses were performed on a portion of the weld bead that solidified first and rejected the thorium into the meit.

Phosphorus was detected as a grain-boundary impurity at low levels in PEF-48 and in substantial quantities in PEF-49. No phosphorus was detected in PEF-44 and PEF-45. Post-test analysis of the plutonia pellets for phosphorus (colorimetric procedure) showed a phosphorus content of $5 \mathrm{ppm}$ in the PEF-44 and PEF-45 pellets, 20 ppm in PEF-48, and 35 ppm in PEF-49. It appears that the phosphorus content of the fuel should be held below $20 \mathrm{ppm}$ to avoid phosphorus pickup by the iridium clad.

\section{B. Second Early Compatibility Test (ECT-2) (D. Pavone)}

The accumulated exposure time for the ECT-2 assembly was 1233 h on March 1, 1983. 


\section{Overpressure Tests (C. Seabourn and R. Zocher)}

The third GPHS overpressure test was completed at $7.4 \mathrm{MPa}$ (1070 psi) in Sandia National Laboratories' 0.57-M shoek tube. The explosion stripped the graphite components from the four $\mathrm{UO}_{2}$, simulant-filled fuel clads, which were recovered intact but lightly deformed.

Simulant-fucled module PAL-001, containing capsules IRG-096, IRG-097, IRG-098, and IRG-099, was stabilized at $1095 \pm 5^{\circ} \mathrm{C}$ in the Sandia shock tube and exposed to a static overpressure of $3.38 \pm 0.34 \mathrm{MPa}$ $(1070 \pm 50 \mathrm{psi})$ and $25.9 \pm 3.5 \mathrm{kPa}=5(3.75 \pm 0.5 \mathrm{psi}+\mathrm{s})$. The explosion stripped the graphite components from the capsules. Fragments of the FWPF components (aeroshell and impact shells) were recovered throughout the vermiculite filling in the cutch tube. No identifiuble piece of the fibrous insulation was found.

Three of the fueled elads were recovered near the rear bulkhead of the catch tube; they hasd penetrated $3.05 \mathrm{~m}$ of vermiculite. The fourth capsule had also penetrated the $19 \mathrm{~mm}$ plywood bukhead and about $100 \mathrm{~mm}$ of the dirt piled behind it. Figure 1 shows where the fourth capsule came to rest. Figure 2 shows the four recovered capsules. The darkest one [Fig. 2(d)] penetrated the plywood and had the greatest nominal diametral strain $(-4.7 \%)$.

Identifying the specfic capsules was impossible, so we sectioned the capsule with the greatest local deformation [Fig. 2(c)] and examined its inner surface. The wrinkles

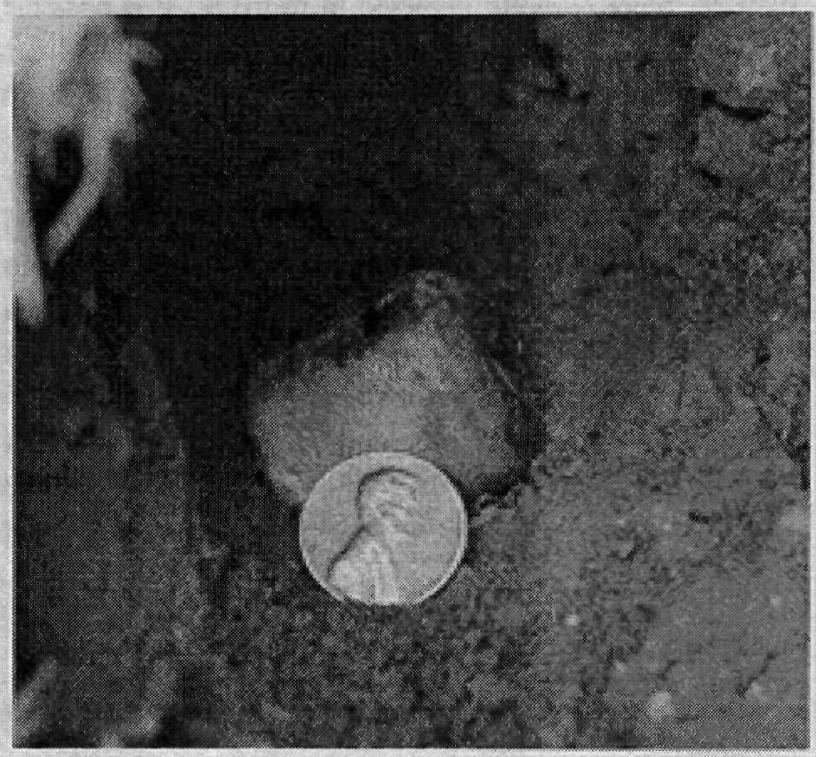

Fig. 1. One capsule in PAL-DOI penctrated the calch-tube rear bolkhesd: ain behind the bukhead gapped the capsule: show that local deformations were fairly sharp, but no cracks occurred (see Fig. 2). Figure 4 shows the $\mathrm{UO}_{2}$ pellet after its removal from the sectioned capsule.

\section{Fragment Tests (C. Seabourn and R. Tate)}

1. Bullet Fragments- The first bullet fragment test was completed. A 50-aliber 18- aluminum-alloy bullet was shot at a half-module simple at $319 \mathrm{~m} / \mathrm{s}^{-1}\left(1048 \mathrm{f} / \mathrm{s}^{-1}\right)$. The bullet penetrated the aeroshell, cracked the impact shell, and deformed the UO, ${ }_{2}$ loaded capsule. The bullet melted where it stopped becanse the module was at $1091^{\circ} \mathrm{C}$, the calculated launch temperature of the heat source. Figure 5 shows a radiograph of the partial module after the test.

2. Plate Frugments. A purchase request was issued to Sandia National Liboratories to conduct the aluminumalloy plate fragment tests. In this test series, 203- by 203by $6.4-\mathrm{mm}$ plates, representing a "standard" fragment from the external fuel ank of the space shuttle, will be propelled at $914 \mathrm{~m} / \mathrm{s}^{-1}\left(3000 \mathrm{f} / \mathrm{s}^{-1}\right)$ into GPHS modules that will have been preheated to $1090^{\circ} \mathrm{C}$. The plates will be mounted on Sundia's rocket sled and will be oriented in the edge-on or face-on direction.

\section{HELIUM RELEASE (D. Peterson)}

The first draft of a Los Alamos report describing the results on the helium release behavior of plutonia was completed this month.

From these results, we calculated the fractional release of helium from a Light-Weight Radioisotope Heater Unit (LWRHU) capsule exposed to $\gamma^{*}$ reentry pulse. The fractional release is shown in Fig, 6 as a function of time and temperature. The predicted release rate is slower than that previously measured. + In prior experiments, the heating pulse was more rapid and extended $100^{\circ} \mathrm{C}$ above the limit of the present calculation. In addition, the fuel used in earlier experiments, which tested vent capabilities, was 8 years old and coarser grained than the production LWRHU fuel used to develop parameters for the calculational model. All differences would be expected to increase the release rate.

fD. E. Peterson and J. S. Starzynski, "Reentry Thermal Testing $\alpha$ Light-Weight Radioisotone Heater Units," Los Alamex National Laboratory report LA-9226 (March 1982). 


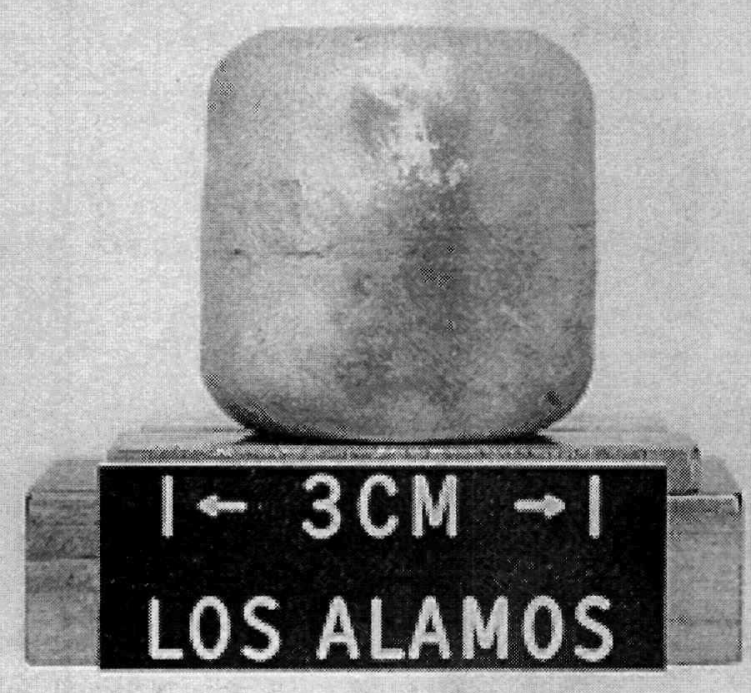

(a)

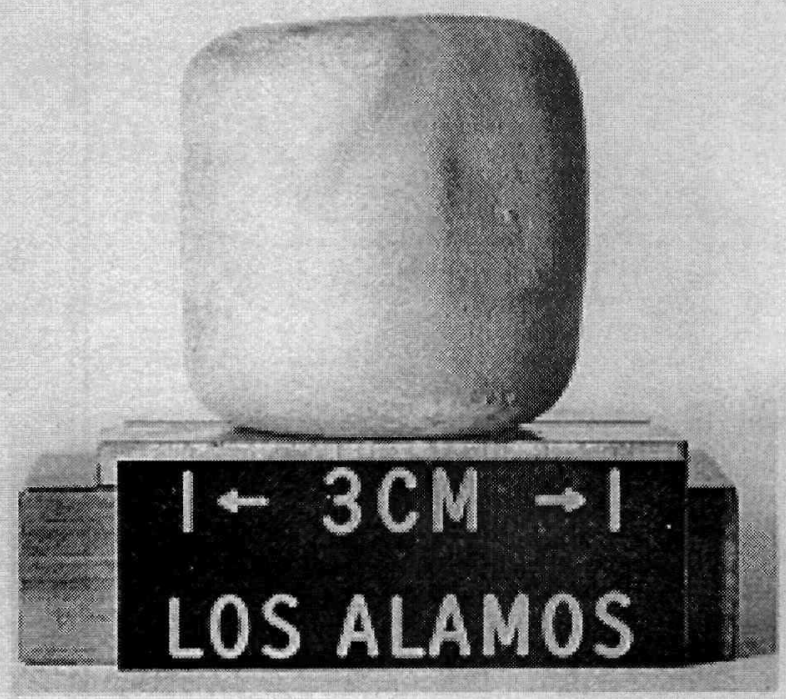

$(\mathrm{c})$

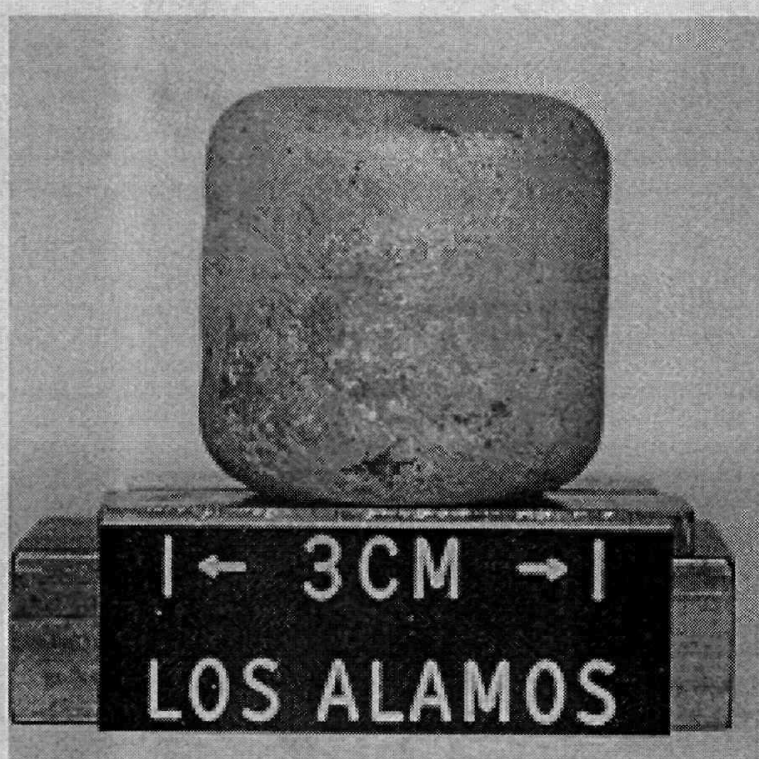

(b)

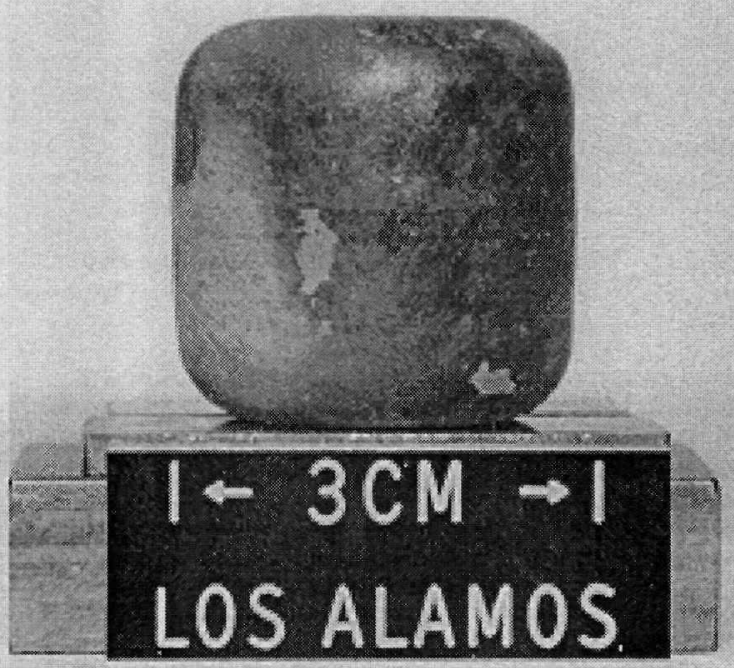

(d)

Fig. 2. The caprodes recovened frem the third overpressure test aere intact. Capsules $2, b$, and c were recowered at the ead of the catch tube. Capsule d peretrated the plowood bulkhead at the end of the tube. 


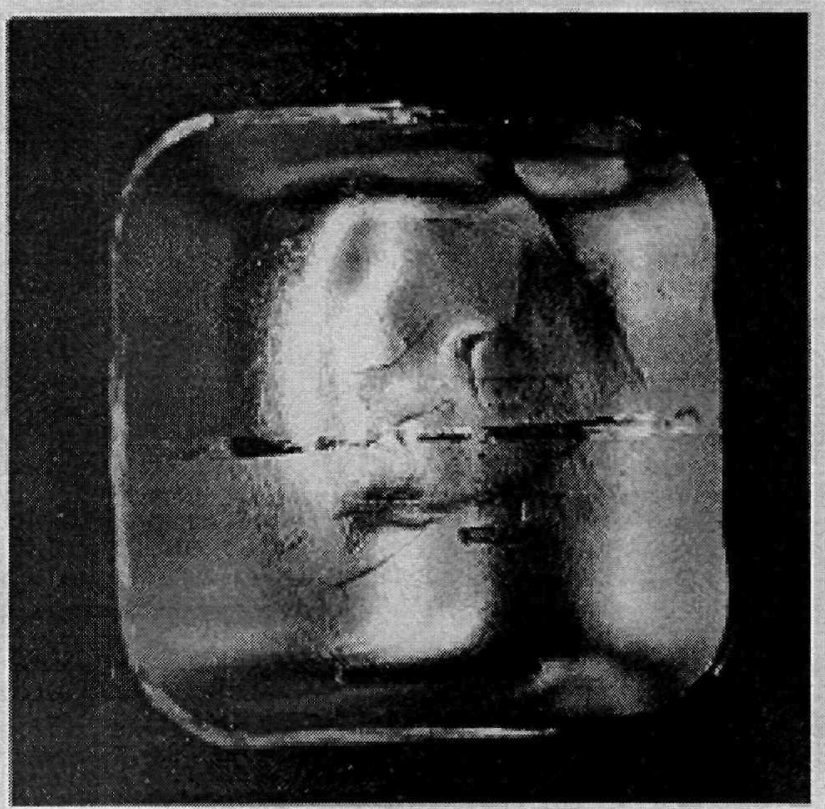

Fig, 3. Cagsule $\mathrm{c}$, with the greatest local deformation, had as eracks $2 \mathrm{X}$

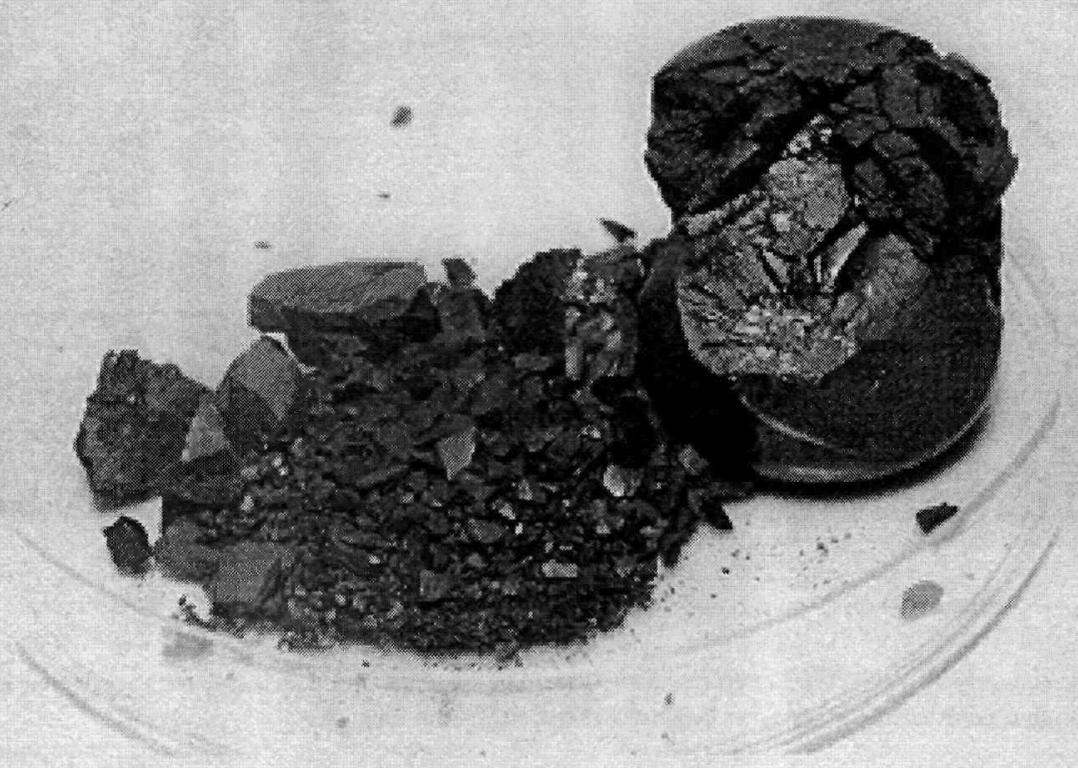

Fig, 4. One end of the $\mathrm{UO}_{2}$ pellat from cxpsule \& was fragmented in the thisd orerpressure test. $1,5 \mathrm{X}$. 


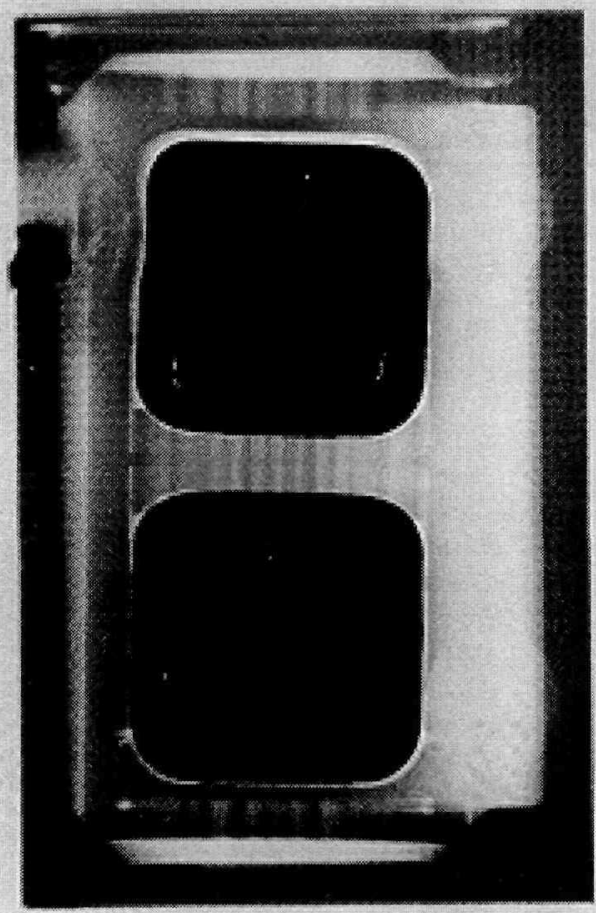

Fig. 5. The $319 \cdot \mathrm{m}^{-1} \mathrm{~s}^{-1}$ aluminum-zloy bulke deformed the target capsule but did not penetrate the impact shell.

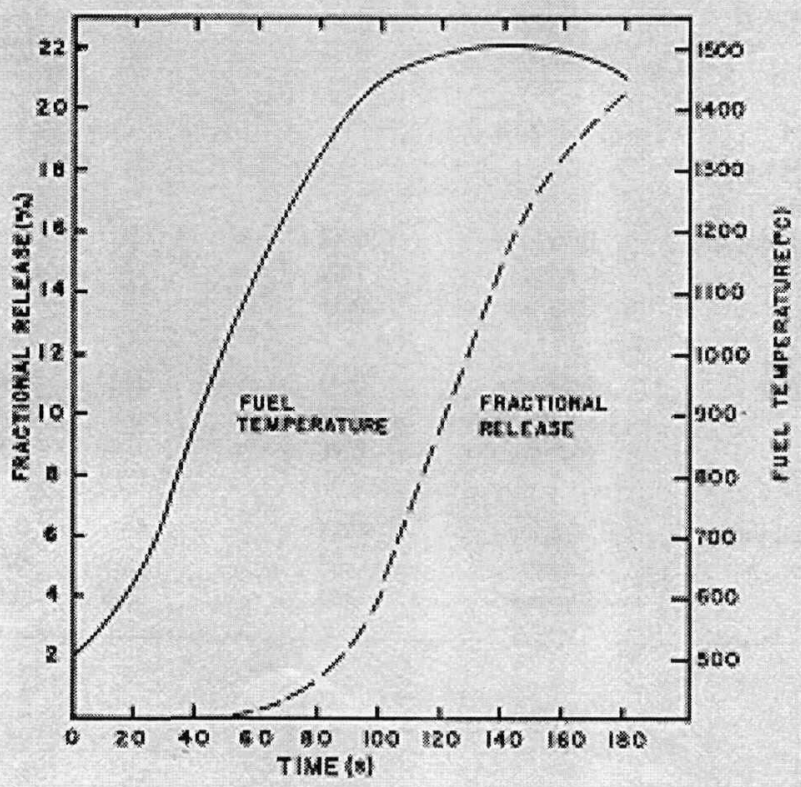

Fig. 6. The helium retesse front a $\mathrm{LWRHU}$ during a $\mathrm{y}^{*}$ reentry heating pulse. 


\begin{tabular}{|c|c|c|c|c|c|c|}
\hline \multirow[b]{2}{*}{ Sample } & \multirow[b]{2}{*}{ Location } & \multicolumn{5}{|c|}{ Intensity Ratios Relative to $\mathbf{I R}_{2}$} \\
\hline & & $\mathrm{TH}_{34}$ & $\mathbf{P}_{120}$. & $\mathrm{C}_{210}$ & $O_{10}$ & Other \\
\hline \multicolumn{7}{|l|}{ Capsule 44} \\
\hline \multicolumn{7}{|l|}{$\begin{array}{l}\text { Cup L256.8 } \\
\text { Archive ting }\end{array}$} \\
\hline \multirow[t]{3}{*}{ As-received } & Interior & 0.80 & --- & - & - & - \\
\hline & Center & 0.66 & -.. & $\ldots$ & - & - \\
\hline & Exterior & 0.80 & -- & -- & -.- & - \\
\hline \multirow[t]{3}{*}{ Aged } & Interior & 0.66 & $\cdots$ & 0.51 & 0.81 & $\ldots$ \\
\hline & Center & 0.87 & - & -- & $\sim$ & - \\
\hline & Exterior & 0.74 & $\cdots$ & 0.49 & $\cdots$ & - \\
\hline \multirow[t]{3}{*}{ Fueled cup } & Interior & 0.33 & $\ldots$ & 0.29 & 0.63 & --- \\
\hline & Center & 0.59 & $\ldots$ & 0.26 & 0.69 &..- \\
\hline & Exterior & 0.43 & $\cdots$ & 0.31 & 0.69 & $--\cdot$ \\
\hline \multicolumn{7}{|l|}{ Cup L254-7 } \\
\hline \multicolumn{7}{|l|}{ Archive ring } \\
\hline As-received & Interior & 0.78 & - & - & $\cdots$ & - \\
\hline & Center & 0.88 & $\cdots$ & -.. & - & --- \\
\hline & Exterior & 0.70 & $\cdots$ & - & - & - \\
\hline \multirow[t]{3}{*}{ Aged } & Interior & 0.44 & - & - & $\cdots$ & - \\
\hline & Center & 0.97 & -.- & - & - & - \\
\hline & Exterior & 0.88 & -- & -- & - & - \\
\hline \multirow[t]{3}{*}{ Fueled cup } & Interior & 0.41 & -- & - & 0.55 & $\cdots$ \\
\hline & Center & 0.74 & - & -- & - & - \\
\hline & Exterior & 0.46 & $\cdots$ & - & 0.42 & $\cdots$ \\
\hline \multirow[t]{3}{*}{ Weld } & Interior & 0.10 & ..- & - & 0.54 & $\cdots$ \\
\hline & Center & 0.51 & $--\cdot$ & - & 1.10 & --- \\
\hline & Exterior & 0.53 & -- & $\cdots$ & 0.82 & $\cdots$ \\
\hline \multicolumn{7}{|l|}{ Capsule 45} \\
\hline \multirow{2}{*}{\multicolumn{7}{|c|}{$\begin{array}{l}\text { Cup L257-R2 } \\
\text { Archive ting }\end{array}$}} \\
\hline & & & & & & \\
\hline \multirow[t]{3}{*}{ As-received } & Interior & 0.82 & $\ldots$ & -.. & - & - \\
\hline & Center & 1.02 & $\ldots$ & $\cdots$ & $\ldots$ & - \\
\hline & Exterior & 0.55 & $\cdots$ & - & -- & - \\
\hline \multirow[t]{3}{*}{ Aged } & Interlor & 0.94 & -- & 0.52 & 0.49 & $\cdots$ \\
\hline & Center & 0.88 & $\ldots$ & 0.23 & 0.40 & $\ldots$ \\
\hline & Exterior & 0.90 & -- & 0.32 & 0.83 & --- \\
\hline \multirow[t]{3}{*}{ Fueled cup } & Interior & 0.24 & -- & - & 0.78 & $\ldots$ \\
\hline & Center & 0.65 & - & - & 0.66 & $\cdots$ \\
\hline & Exterior & 0.57 & -- & 0.40 & 0.90 & -- \\
\hline
\end{tabular}


TABLE I. (cont)

\begin{tabular}{|c|c|c|c|c|c|c|}
\hline \multirow[b]{2}{*}{ Sample } & \multirow[b]{2}{*}{ Location } & \multicolumn{5}{|c|}{ Intensity Ratios Relative to $\mathbf{I R}_{229}$} \\
\hline & & $\mathrm{TH}_{94}$ & $\mathbf{P}_{120}$ & $\mathrm{C}_{370}$ & $\mathbf{O}_{310}$ & Other \\
\hline \multicolumn{7}{|l|}{ Cup 255-6 } \\
\hline \multicolumn{7}{|l|}{ Archive ring } \\
\hline \multirow[t]{3}{*}{ As-received } & Interior & 0.80 & $\cdots$ & --- & $\ldots$ & - \\
\hline & Center & 0.96 & $\ldots$ & $\cdots$ & - & - \\
\hline & Exterior & 0.87 & -.- & $\cdots$ & -- &.- \\
\hline \multirow[t]{3}{*}{ Aged } & Interior & 0.71 & $\cdots$ & $\cdots$ & --- & $\cdots$ \\
\hline & Center & 1.03 & $\ldots$ & $\cdots$ & - & $\cdots$ \\
\hline & Exterior & 0.66 & -- & 0.42 & 0.59 & $\cdots$ \\
\hline \multirow{3}{*}{ Fueled cup } & Interior & 0.31 & -- & ..- & 0.47 & -- \\
\hline & Center & 0.69 & ..- & $\ldots$ & - & - \\
\hline & Exterior & 0.27 & --- & - & 0.59 & -- \\
\hline \multirow[t]{3}{*}{ Weid } & Interior & 0.11 & $\cdots$ & ... & -- & - \\
\hline & Center & 0.42 & $\cdots$ & $\ldots$ & 0.61 & ... \\
\hline & Exterior & 0.40 & - & 0.40 & 1.00 & ... \\
\hline
\end{tabular}

Capsule 48

Cup N502-3

Archive ring

As-recelved

Interior

Center

Exterior

Aged

Interior

Center

Exterior

Fueled cup

Interior

Exterior

Cup P701-4

Archive ring

As-received

Interior

Center

Exterior

Aged

Interior

Center

Exterior

Fueled cup

Weld

Interior

Center

Exterior

Interior

Center

Exterior $\begin{array}{lllll}0.60 & - & -- & - & - \\ 0.91 & - & -- & - & - \\ 0.64 & \ldots & \ldots & \ldots & --\end{array}$

$\begin{array}{lllll}0.84 & - & 0.82 & 1.03 & 0.07(\mathrm{~S})\end{array}$

$0.18(\mathrm{~B}$ or $\mathrm{Cl})$

$\begin{array}{llll}0.15 & 0.64 & 0.97 & 0.09(\mathrm{~S})\end{array}$

$\begin{array}{lllll}0.82 & - & 0.75 & 1.28 & 0.19(\mathrm{~B} \text { or } \mathrm{Cl})\end{array}$

$\begin{array}{llllllllll}0.61 & \ldots & \ldots & 0.51 & \ldots\end{array}$

$\begin{array}{lllll}0.49 & 0.26 & -- & \ldots & - \\ 0.68 & 0.36 & \ldots- & 0.67 & --\end{array}$

Energy of observed peak slightly shitted. 


\begin{tabular}{|c|c|c|c|c|c|c|}
\hline \multirow[b]{2}{*}{ Sample } & \multirow[b]{2}{*}{ Location } & \multicolumn{5}{|c|}{ Intensity Ratios Relative to IR $_{219}$} \\
\hline & & $\mathbf{T H}_{14}$ & $\mathbf{P}_{12 a}$ & $\mathrm{C}_{270}$ & $\mathbf{O}_{s 10}$ & Other \\
\hline \multicolumn{7}{|l|}{ Capsule 49 } \\
\hline \multicolumn{7}{|l|}{$\begin{array}{l}\text { Cup PR715-2 } \\
\text { Archive ring }\end{array}$} \\
\hline \multirow[t]{3}{*}{ As-received } & Interior & 0.74 & ..- & - & -- & - \\
\hline & Center & 0.84 & $\cdots$ & ... & - & - \\
\hline & Exterior & 0.66 & $\cdots$ & $\cdots$ & $\cdots$ & - \\
\hline \multirow[t]{3}{*}{ Aged } & Interior & 0.85 & $\ldots$ & - & - & - \\
\hline & Center & 3.01 & -- & $\ldots$ & - & - \\
\hline & Exterior & 0.81 & $\cdots$ & $\ldots$ & - & - \\
\hline \multirow[t]{3}{*}{ Fueled cup } & Interior & 0.56 & 0.16 & ... & $\ldots$ & - \\
\hline & Center & 0.61 & 0.15 & $\ldots$ & - & ... \\
\hline & Exterior & 0.77 & 0.32 & .-- & $\ldots$ & - \\
\hline \multicolumn{7}{|l|}{$\begin{array}{l}\text { Cup P714-5 } \\
\text { Archive ring }\end{array}$} \\
\hline \multirow[t]{3}{*}{ As-received } & Interior & 0.61 & $\ldots$ & -.. & 0.65 & -.. \\
\hline & Center & 0.68 & --- & $\ldots$ & -- & - \\
\hline & Exterior & 0.73 & -- & -- & 0.74 & ... \\
\hline \multirow[t]{3}{*}{ Aged } & Interior & 0.72 & $\ldots$ & -- & - & $\ldots$ \\
\hline & Center & 0.71 & $\ldots$ & ... & - & - \\
\hline & Exterior & 0.87 & ... & ... & - & - \\
\hline \multirow[t]{3}{*}{ Fueled cup } & Interior & 0.21 & 1.29 & $\cdots$ &.. & $\ldots$ \\
\hline & Center & 0.64 & 0.23 & $\ldots$ & - & - \\
\hline & Exterior & 0.46 & 0.26 & $\cdots$ & $\cdots$ & $0.26(\mathrm{Si})$ \\
\hline \multirow[t]{3}{*}{ Weld } & Interior & $\ldots$ & -.. & 0.71 & 1.16 & $0.14(S)$ \\
\hline & Center & --- & ... & 0.33 & 0.60 & $\cdots$ \\
\hline & Exterior & 0.13 & .. & 0.64 & 0.80 & $0.05(\mathbf{S})$ \\
\hline \multicolumn{7}{|l|}{ Capsule T40 } \\
\hline \multicolumn{7}{|l|}{$\begin{array}{l}\text { Cup NR520-2 } \\
\text { Archive ring }\end{array}$} \\
\hline \multirow[t]{3}{*}{ As-received } & Interior & 0.89 & $\ldots$ &..- & - & - \\
\hline & Center & 0.78 & - & $\ldots$ & $\ldots$ & .-- \\
\hline & Exterior & 0.71 & - & $\cdots$ & $\cdots$ & $\cdots$ \\
\hline \multirow[t]{3}{*}{ Aged } & Interior & 0.75 & -- & -. & $\ldots$ & - \\
\hline & Center & 0.85 & ... &.-- & - & - \\
\hline & Exterior & $\delta .71$ & $\ldots$ & 0.90 & 0.67 & -.- \\
\hline \multicolumn{7}{|l|}{ Unfueled cup } \\
\hline \multirow[t]{3}{*}{ Aged } & Interior & 0.73 & $\ldots$ & 0.23 & 0.37 & -.- \\
\hline & Center & 0.88 & $\ldots$ & .. & --- & $\ldots$ \\
\hline & Exterior & 0.77 & - & - & 0.46 & -- \\
\hline
\end{tabular}




\begin{tabular}{|c|c|c|c|c|c|c|}
\hline \multirow[b]{2}{*}{ Sample } & \multirow[b]{2}{*}{ Location } & \multicolumn{5}{|c|}{ Intensity Ratios Relative to $\mathbf{I R}_{219}$} \\
\hline & & $\mathrm{TH}_{34}$ & $\mathbf{P}_{120}$ & $\mathrm{C}_{270}$ & $\mathrm{O}_{\mathrm{si0}}$ & Other \\
\hline \multicolumn{7}{|l|}{ Cup P719-6 } \\
\hline \multirow[t]{3}{*}{ As-received } & Interior & 0.91 & $--\cdot$ & $\cdots$ & $\cdots$ & --- \\
\hline & Center & 0.94 & --- & --- & -- & -- \\
\hline & Exterior & 0.65 & -- & $\cdots$ & 0.56 & -- \\
\hline \multirow[t]{3}{*}{ Aged } & Interior & 0.97 & -- & -- & --- & -.. \\
\hline & Center & 1.13 & --- & --- & $--\cdot$ & -- \\
\hline & Exterior & 0.84 & $\cdots$ & ..- & 0.82 & $\cdots$ \\
\hline \multicolumn{7}{|l|}{ Unfuteled cup } \\
\hline \multirow[t]{3}{*}{ Aged } & Interior & 0.75 & -- & -- & 0.50 & -- \\
\hline & Center & 0.74 &..- & -.- & $\cdots$ & $\ldots$ \\
\hline & Exterior & 0.67 & -- & $\cdots$ & 0.39 & $\cdots$ \\
\hline \multirow[t]{3}{*}{ Weld } & Interior & 0.61 & $\cdots$ & 0.87 & 0.64 & -- \\
\hline & Center & 0.72 & $\cdots$ & 0.39 & 0.61 & -- \\
\hline & Exterior & 0.73 & $\cdots$ & 2.37 & 1.15 & $\cdots$ \\
\hline
\end{tabular}




\section{ADDITIONAL DISTRIBUTION}

B. J. Rock, Dept. of Energy/OSNP, Washington, DC

G. L. Bennett. Dept. of Energy/OSNP, Washington, DC

J. J. Lombardo, Dept. of Energy/OSNP, Washington, DC

R. B. Morrow, Dept. of Energy/OSNP, Washington, DC

R. Brouns. Dept. of Energy/OSNP, Washington, DC

J. Griffo, Dept. of Energy/OSNP, Washington, DC

D. K. Stevens, Dept. of Energy/BES, Washington, DC

M. Norin, Dept. of Energy, W ashington, DC

G. Ogburn, Dept. of Energy, Washington, DC

J. A. Yoder, Dept. of Energy, Washington, DC

C. Osterberg, Dept, of Energy, Washington, DC

1. Van Der Hoven, Dept. of Energy, Washington, DC

R. L. Clark, Dept. of Energy/ALO, Albuquerque, NM

J. P. Crane, Dept. of Energy, Albuquerque, NM

K. Elliot, Dept. of Energy. Albuquerque, NM

D. L. Krenz, Dept. of Energy, Albuqquerque, NM

J. R. Roeder, Dept. of Energy, Albuquerque, NM

R. B. Crouch, Dept. of Energy, Albuquerque, NM

J. N. Bailey, Dept. of Energy, Albuquerque, NM

H. N Hitl, Dept. of Energy/DOA, Miamisburg, OH

L. C. Sjostrom, Dept. of Energy, Aiken, SC

R. J. Hart, Dept. of Energy/ORO, Oak Ridge, TN

J. Pidkowicz, Dept. of Energy, Oak Ridge, TN

W. L. Von Flue, Dept. of Energy, SFOO, Oakland, CA

T. B. Kerr, NASA, Washington, DC

F. R. Sehmidt, NASA, Washington, DC

E. Gabris, NASA. Washington, DC

N. Sculze. NASA, Washington, DC

B. R. McCullar, NASA, Washington, DC

Operations and Systems Requirements, NASA, Washington, DC

A. v. Diaz, NASA, Washington, DC

R. G. Ivanoff, NASA/IPL, Pasadena, CA

R. Campbell, NASA/JPL, Pasadena, CA

L. T. Shaw. JPL, Pasadena, CA

R. J. Spehalski, JPL, Pasadena, CA

AFISC/SNS, Attn: Col. J. A. Richardson, Kirtland AFB, NM

AFISC/NTYNS, Attn: Capt. D. K. Zimmerman, Kirtand AFB, NM

AFWL/NTYNS, Atn: Maj. J. D. Martens, Kirtland AFB, NM

Lt. Col. James H. Lee, Jr., AFWL/NTYN, Kirtland AFB, NM

Capt. Michael K. Seaton. AFWL/NTYNP, Kirtland AFB, NM

Maj. Ray Baca, HQ AFSC/IGF, Andrews AFB, Washington, DC

Col. William Licht, ATSD/AE, Washington, DC

L.t. John Erb, ESMC/SGPH, Patrlek AFB, FL.

L. J. Ullian. ESMC/SE. Patrick AFB, FL

D. W. Keesler, AFML/MPE, Wright-Patterson AFB, OH

Lt. Col. Mel Nosal, AFISC/SES, Norton AFB, CA

Lt. Col. Ken Morrison, AFISC/SES, Norton AFB, CA

Maj. Walt Wilson, AFISC/SEM, Norton AFB, CA

John Marshall, AFRPL/LKC, Edwards AFB, CA

L. Col. Willtams B. Moyer, AFMSC/SGPZ, Brooks AFB, TX

W. Riley, WSMC/SE, Vandenberg AFB, CA

Capt. Chartes Snow, SAMSO/SE, Los Angeles, CA

HQ Space Div./YLVS, Attr: Lt. Col. Neectham, Los Angeles, CA

R. L. Folger, SRL, Aiken, SC

J. Howell, SRL, Aiken, SC

J. B. Mellen, SRP, Aiken, SC

G. L. Albert, SRP Aiken, SC

B. Roberts, SRP, Aiken, SC

J. R. MeClain, MRC, Miamisburg, OH

W. T. Cave, MRC, Miamisburg. OH

W. Amos. MRC. Miamisburg, OH
E. W. Johnson, MRC, Miamisburg, OH

R. Cooper, ORNL, Oak Ridge, TN

C. Alexander, BCL, Columbus, $\mathrm{OH}$

E. E. Rice, BCL, Columbus, $\mathrm{OH}$

J. Hagan, APL, Baltimore, MD

B. Bartram, NUS Corporation, Gaithersburg, MD

R. W. Engleharl, NUS Corporation, Oaithersburg, MD

H. H. Van Tuyl, PNL, Richland, WA

A. Schock, Fairchild-Hiller Ind., Germantown, MD

E. Skrabek, Fairchild-Hiller Ind., Gęrmantown, MD

C. W. Whitmore, GE, Philadelphia, PA

C. T. Bradshaw, GE, Philadelphia, PA

R, Kelley, GE, Philadelphia, PA

R. Hemler, GE, Philadelphia, PA

Y. Haley, GE, Phlladeiphia, PA

R. Hartman, GE, Philadelphia, PA

W. Mecham, ANL, Argonne, IL

D. C. Anderson, Teledyne Energy Systems, Timonium, MD

P. Dick, Teledyne Energy Systems, Timonium, MD

Dr. Marvin Goldman, University of California, Davis, CA

Charles Smith, Sandia Nationa Laboratories, Albuquerque, NM

R. Harner, Sandia National Laboratories, Albuquerque, NM

C. M. Barnes, L. B. Johnson Space Center, NASA SPS, Houston, TX

R. H. Brown, L. B. Johnson Space Center, NASA FM, Houston, TX

R. G. Rose, L. B. Johnson Space Center, NASA FA, Houston, TX

Harold Battaglia, L. B. Johnson Space Center, NASA PF, Houstan, TX.

W. H. Boggs, NASA, DE-A, J. F. Kennedy Space Center, FL

Lloyd Parker, NASA, SF, J. F. Kennedy Space Center, FL

George M. Marmaro, NASA, MD.ESB, J. F. Kennedy Space Center, FL

W. A. Riehl, Marshall Space Flight Center, NASA, EH3I, Marshall SFC, AL

w. C. Pitts, NASA, STPM, Ames Research Center, Moffett Fiejd, CA

J. J. Givens, Ames Research Center, Moffett Field, CA

R. Corriden, Ames Research Center-NASA, Moffett Field, CA

J. C. Robinson, Lengley Resesrch Center, NASA, MS395, Hampton, VA

G. J. Schaefer, Jr., Lewis Research Center, NASA, MAIC 500-120,

Cleveland, $\mathrm{OH}$

R. C. Turkolu, TRW, Defense and Space Systems Group, Redonds Beach, CA

D. Eaton, European Space Research and Technology Centre, Zw'arteweg 62, Njordwijk, The Netherlands

Dr. Ralph R. Fullwood, Science Applications, Inc., Palo Alto, CA Dr. William Ailor, The Aeronpace Corporation, Los Angeles. CA T. Carter, Nuclear Regulatory Commission. Washington, DC

C. R. Chappell, Nuclear Regulatory Cummission, Washington, DC

D. Eggan, Ofice of Radiation Programs, USEPA, Washington, DC

$\mathrm{N}$. Elsner, General Atomics, San Diego, CA

Charles Salisbury, NavaI Ocean Systems Center, San Diego, CA

Dr. Herbert Weiss, Naval Ocean Systems Center, San Diego, CA

W. J. Maraman, Los Alamos National Laboratory, Los Alamos, NM

S. S. Hecker, Los Alamos National Laboratory, Los Alamos, NM

R. N. R. Mulford, Los Alamos National Laboratory, Los Alamos, NM

J. Bircly, Los Alamos Nationai Laboratory, Los Alamos, NM

W. F. Miller, Los Alamos, National Laboratory, Los Alamos, NM

R. J. Pryor, Los Alamos National Laboratory, Los Alamos, NM

S. E. Bronisz, Los Alamos National Laboratory, Los Alamos, NM

w. Stark, Los Alamos National Laboratory, Los Alamos, NM

R. W. Zocher, Los Alamos National Laboratory, Los Alamos, NM

J. A. Pattillo, Los Alamos National Laboratory, Los Alamos, NM

E. M. Wewerka, Los Alamos National Laboratory, Los Alamos, NM

T. K. Keenan, Los Alamos Natonal Laboratory, Los Alamos, NM 